\title{
Forma Isolada do Miocárdio Não-Compactado
}

\author{
Isolated Noncompaction of the Myocardium
}

Dinaldo Cavalcanti de Oliveira, Marcelo Menezes Malta, Jairo Alves Pinheiro, Leopoldo Soares Piegas

Hospital do Coração - Hcor - São Paulo, SP

Miocárdio não-compactado (MNC) é uma cardiopatia congênita com incidência rara, sendo o seu primeiro relato feito há 15 anos e com poucos casos publicados. O objetivo deste artigo é descrever um caso de MNC. É apresentada descrição dos achados clínicos e dos exames complementares de imagem de uma paciente com 37 anos, portadora de MNC de forma isolada. A paciente queixava-se de palpitações, apresentava extra-sístoles no exame clínico e, no eletrocardiograma de 12 derivações, bigeminismo ventricular. Realizou ecocardiograma Doppler tridimensional que revelou a presença de numerosas e proeminentes trabéculas com recessos intertrabeculares profundos com fluxo de sangue que se comunicavam com a cavidade ventricular e que se acentuavam na região septoapical. A ressonância nuclear magnética de coração corroborou os achados do ecocardiograma. A clínica e os resultados dos exames complementares dessa paciente confirmaram o diagnóstico de $\mathrm{MNC}$ de forma isolada. O conhecimento de achados ecocardiográficos dessa doença permite um diagnóstico precoce e tratamento mais adequado.
Noncompacted myocardium (NCM) is a rare congenital heart disease, first reported 15 years ago and with only a few published cases. In this paper, we report the main clinical findings and the complementary exams that suports NCM diagnostic. Discretion of anamnesis and physical examination, together with characteristic image complementary exams findings of symptomatic NCM. (Case report). The clinical assessment and the electrocardiogram at the admission found bigeminism ventricular. A tridimensional Doppler echocardiogram was performed and showed numerous and prominent myocardium trabeculations and deep intertrabecular recess filled with blood that communicate with the ventricular cavity, more present in the septal-apical area. Magnetic resonance imaging supported the echocardiographic findings and ruled out the presence of others cardiac malformations. The clinical and imaging complementary exams filled out the touchstones that support isolated NCM diagnose. Is important to know that suggestive NCM findings permits an earlier diagnostic and treatment.

\section{Introdução}

Durante o desenvolvimento embriológico normal, em uma fase inicial, o coração apresenta aspecto poroso em razão de uma rede de fibras entrelaçadas que formam trabéculas separadas por recessos profundos que se comunicam com a cavidade ventricular ${ }^{1}$.

Entre a $5^{\mathrm{a}}$ e a $8^{\mathrm{a}}$ semanas de vida embrionária, esse miocárdio sofre compactação no sentido do epicárdio para o endocárdio e da base para o ápice, o que determinará o aspecto normal do músculo cardíaco. A circulação coronariana se desenvolve durante esse processo e os recessos intertrabeculares são reduzidos a capilares. Essa compactação ocorre de forma mais importante no ventrículo esquerdo que no direito ${ }^{2,3}$.

Miocárdio não-compactado (MNC) é uma cardiopatia congênita rara, descrita inicialmente por meio de necrópsia em 1932. Na década de 1980, surgiram as primeiras publicações que utilizaram critérios ecocardiográficos para diagnóstico dessa enfermidade ${ }^{4}$. A falha no processo

\section{Palavras-chave}

Cardiopatias congênitas, coração / embriologia, disfunção ventricular.

\section{Correspondência: Dinaldo Cavalcanti de Oliveira •}

Av. Eliseu Guilherme, 123

04040-030 - São Paulo, SP

E-mail: doliveira@hcor.com.br

Artigo recebido em 05/03/06; revisado recebido em 02/04/06; aceito em 12/06/06. de compactação do miocárdio resulta na persistência de trabeculações e recessos profundos que se comunicam com a cavidade ventricular, manifestação característica dessa doença. O ventrículo esquerdo é sempre acometido, sendo ambos os ventrículos envolvidos em menos de $50 \%$ dos casos, com predominância apical ${ }^{1}$.

De acordo com critérios ecocardiográficos, a prevalência é de 0,014\%; entretanto, é possível que esses números estejam subestimados, pois a maioria dos estudos foi realizada em hospitais terciários e em pacientes sintomáticos². Os homens são mais acometidos do que as mulheres, podendo haver ocorrência familiar em até $44 \%$ dos $\operatorname{casos}^{1,3,5}$.

A seguir, descreveremos os principais achados clínicos e dos exames complementares de uma jovem paciente com miocárdio não-compactado isolado, de acordo com critérios ecocardiográficos.

\section{Relato do Caso}

Paciente do sexo feminino, 37 anos, médica, branca, solteira, católica, natural de Barra Mansa (Rio de Janeiro) e procedente de São Paulo, foi internada no dia 27 de outubro de 2005 com queixa de palpitações intermitentes havia duas semanas. Na avaliação cardiológica prévia durante um desses períodos de palpitações foi identificada taquicardia paroxística supraventricular (TPSV) revertida com manobras vagais. Negava tabagismo, etilismo ou antecedentes familiares relevantes. O exame físico era compatível com a normalidade, exceto pela presença de extra-sístoles freqüentes. 


\section{Relato de Caso}

Realizou eletrocardiograma de 12 derivações em repouso, que evidenciava bigeminismo ventricular; radiografias de tórax (incidências póstero-anterior e perfil esquerdo) que não apresentavam anormalidades; ecocardiograma transtorácico tridimensional que demonstrou ausência de outras anormalidades cardíacas, proeminentes e numerosas trabéculas com recessos intertrabeculares profundos; e ao Doppler colorido observou-se fluxo de sangue nesses recessos com comunicação com a cavidade ventricular. Essas trabeculações eram mais numerosas e proeminentes em região septo-apical do ventrículo esquerdo (fig. 1). Também foi diagnosticada disfunção diastólica do ventrículo esquerdo (VE). A paciente foi submetida a ressonância nuclear magnética (RNM) cujos achados foram concordantes com os do ecocardiograma (fig. 2).

De acordo com critérios clínicos e com os resultados dos exames complementares, diagnosticou-se miocárdio não- compactado de forma isolada. Administrou-se amiodarona em doses habituais com manutenção do ritmo sinusal e ausência de extra-sístoles ventriculares.

Como complemento da estratificação do risco cardiovascular a paciente foi submetida a estudo eletrofisiológico (EEF) e teste ergométrico, que foram normais.

No dia 8 de novembro de 2005, a paciente teve alta hospitalar, em boas condições clínicas, assintomática e orientada a repetir o EEF após um ano, em razão do risco de morte súbita.

A paciente foi orientada a pedir que todos os seus parentes de primeiro grau realizassem avaliação cardiológica e ecocardiograma bidimensional com Doppler.

S.A.C está em acompanhamento ambulatorial há três meses e evolui assintomática.

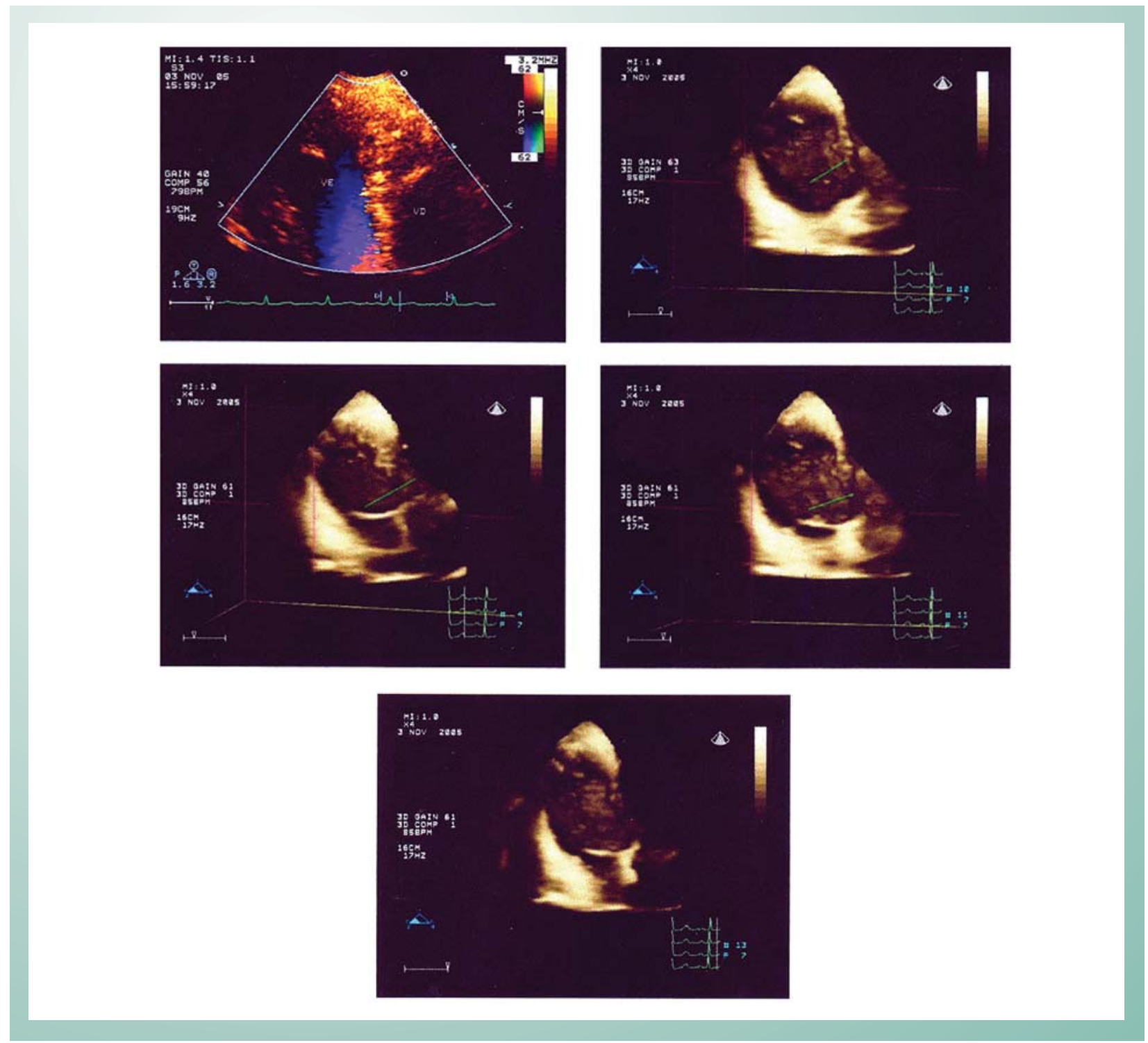

Fig. 1 - Ecocardiograma revelando trabeculações e recessos no miocárdio. 


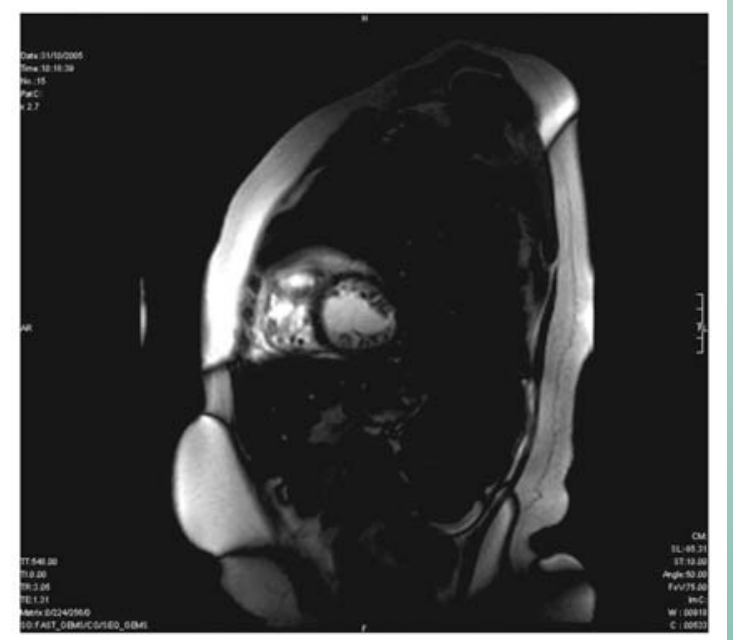

Fig. 2 - Ressonância magnética: aumento das trabeculações no miocárdio.

\section{Discussão}

A primeira descrição do $\mathrm{MNC}$ foi em associação com outras cardiopatias congênitas (origem anômala da coronária esquerda do tronco da artéria pulmonar, obstrução da via de saída dos ventrículos esquerdo e direito) ${ }^{1}$.

Acredita-se que a não-compactação se deva ao fato de os ventrículos estarem expostos a pressões muito elevadas durante a vida intra-uterina. MNC de forma isolada é ainda mais raro e sua causa, não bem esclarecida ${ }^{1,6,7}$.

Formas esporádicas e familiares de MNC têm sido descritas. Em estudos que envolvem MNC isolada com predominância de crianças, a ocorrência familiar foi vista em metade dos pacientes. Estudos do MNC isolado em adultos revelam recorrência familiar de 18\%. Apesar de os genes responsáveis pelas formas esporádicas não terem sido identificados, em casos familiares tem sido descrita uma série de genes relacionados à enfermidade ${ }^{2,5}$.

Os pacientes podem permanecer assintomáticos durante toda vida ou apresentar mais comumente as seguintes manifestações clínicas: disfunção sistólica do VE, que surge por disfunção da microcirculação e pela hipoperfusão subendocárdica, em razão do aumento da demanda de oxigênio determinada pela contração isométrica no endocárdio e miocárdio que se continuam nos recessos intertrabeculares; disfunção diastólica do VE, causada tanto por alteração de relaxamento como por restrição diastólica provocada pelas numerosas trabéculas; arritmias cardíacas, tais como fibrilação atrial e ventricular; bloqueios atrioventriculares ou de ramo; síndrome de WolfParkinson-White e complicações embólicas, decorrentes do trombo nos átrios ou nos recessos intertrabeculares ${ }^{1,2,7,8}$.

O ecocardiograma com Doppler é o exame complementar mais importante no diagnóstico do MNC. Os critérios usados para o diagnóstico são: 1. ausência de anomalias cardíacas, assim como nas valvas aórticas e pulmonares ou nas artérias coronárias; 2 . proeminentes e numerosas trabéculas com recessos intertrabeculares profundos e razão do espessamento entre as camadas compactada e não-compactada $>2 ; 3$. identificação de fluxo sangüíneo diretamente da cavidade do ventrículo dentro dos recessos intertrabeculares; 4 . envolvimento especialmente das regiões lateral média, inferior média ou apical de VE. O MNC na sua forma isolada é

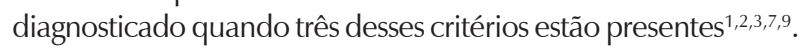
O ventrículo esquerdo é sempre afetado e o acometimento biventricular ocorre em menos de $50 \%$ dos $\operatorname{casos}^{1,2,10}$.

A paciente em questão apresentou palpitações, sendo diagnosticada TPSV e bigeminismo ventricular. Pelo ecocardiograma, constatou-se disfunção diastólica, o que possivelmente contribuiu para origem do bigeminismo e para o aumento da pressão no átrio esquerdo, que pode determinar o surgimento de arritmias supraventriculares. Os achados ecocardiográficos da paciente foram típicos da MNC, e como os quatro critérios descritos anteriormente estavam presentes, caracterizou-se sua forma isolada.

Em dois outros relatos de casos de MNC, a RNM foi utilizada para confirmação do diagnóstico ${ }^{11,12}$. Este exame de imagem pode ser utilizado no diagnóstico e fornece evidências convincentes em casos em que os achados ecocardiográficos são duvidosos. Testes genéticos para mutações conhecidas podem fornecer dados adicionais para aconselhamento e pesquisa $^{13-16}$.

Diagnóstico diferencial inclui: proeminência de trabeculações miocárdicas normais $(<3)$, falsos tendões e bandas aberrantes, tumores cardíacos e trombo apical de VE, cardiomiopatia hipertrófica apical, miocardiopatia dilatada, displasia arritmogênica do ventrículo direito e fibroelastose endocárdica ${ }^{1,2,14,17}$.

O tratamento depende da apresentação clínica. Nos casos de insuficiência cardíaca congestiva, o tratamento é realizado de forma semelhante às de outras causas. Alguns autores, pelo risco de morte súbita, sugerem realização de estudo eletrofisiológico e Holter de 24 horas na avaliação inicial do paciente, e anualmente. Indica-se anticoagulação para todos os pacientes, independentemente da identificação de trombo intracardíaco. Os esportes competitivos são proscritos e a gravidez deve ser evitada ${ }^{2,4,13,17}$.

O prognóstico varia amplamente, desde casos em que os pacientes permanecem assintomáticos durante toda vida até casos de rápida deterioração da função cardíaca com óbito precoce $^{1,2,18}$.

Parentes de primeiro grau de pacientes com MNC devem realizar ecocardiograma com Doppler como exame de triagem $^{1,2,4}$. Tal recomendação foi feita à paciente, pois o diagnóstico precoce permite melhor estratificação de risco (incluindo de morte súbita) e tratamento.

Concluímos que o conhecimento dos achados ecocardiográficos permite o diagnóstico precoce e melhor tratamento do MNC.

\section{Potencial Conflito de Interesses}

Declaro não haver conflitos de interesses pertinentes. 


\section{Relato de Caso}

\section{Referências}

1. Corrado G, Santarone M, Miglierina E, Beretta S, Frattini T, Tadeo G. Isolated noncompaction of the ventricular myocardium. A study in an adult male and literature review. Ital Heart J. 2000; 1: 372-5.

2. Weiford BC, Subbarao VD, Mulhern KM. Noncompaction of the ventricular myocardium. Circulation. 2004; 109: 2965-71

3. Ritter M, Oechslin E, Sutsch G, Attenhofer C, Schneider J, Jenni R. Isolated noncompaction of the myocardium in adults. Mayo Clin Proc. 1997; 72: 26-31

4. Gimenes VML. Cardiomiopatias restritivas e infiltrativas. In: Nobre F Jr Serrano CV. (eds). Tratado de cardiologia. São Paulo: SOCESP/Manole; 2005. p. 879-80.

5. Bleyl SB, Mumford BR, Brown-Harrison MC, Pagotto LT, Carrey JC, Pysher TJ. Xq28-linked noncompaction of the ventricular myocardium: prenatal diagnosis and pathologic analysis of affected individuals. Am J Med Genet. 1997; 72: 257-65

6. Dusek J, Bohuslav O, Duskova M. Posnatal persistence of spongy myocardium with embrionicbblood supply. Arch Pathol. 1975; 99: 312-7.

7. Chin TK, Perloff JK, Williams RG, Jue K, Mohrmann R. Isolalated noncompaction of left ventricular myocardium: a study of eight cases. Circulation. 1990; 82: 507-13.

8. Soler R, Rodriguez E, Monserrat L, Alvarez N. Magnetic resonance imaging of subendocardial perfusion deficits in isolated left ventricular noncompaction. J Comput Assist Tomogr. 2002; 26: 373-5

9. Giafana P, Badano LP, Faganello G, Tosoratti E, Fioretti PM. Additive value of contrast echocardiography for the diagnosis of noncompaction of the left ventricular myocardium. Eur J Echocardiogr. 2006; 7: 67-70.

10. Jenni R, Oechslin E, Schneider J, Attenhofer Jost C, Kaufmann PA.
Echocardiographic and pathoanatomical characteristics of isolated left ventricular non-compaction: a step towards classification as a distinct cardiomyopathy. Heart. 2001; 86: 666-71.

11. Salemi VMC, Araujo AQ, Arteaga E, Mady C. Pitfalls in the echocardiographic diagnosis of isolated non-compaction of the ventricular. Heart. 2005; 11 1382.

12. Salemi VMC, Rochitte CE, Lemos P, Benvenuti LA, Pita CG, Mady C. Longterm survival of a patient with isolated noncompaction of the ventricular myocardium. J Am Soc Echocardiogr. 2006; 3: 354.e1-354.e3.

13. Botto LD. Left ventricular nomcompaction. Orphanet encyclopedia [citado em 2006 fevereiro 10]. Disponível em: http://www.orpha.net/data/patho/ GB/uk-LVNC.pdf/.

14. Hamamichi Y, Ichida F, Hashimoto I, Uese KH, Miyawaki T, Tsukano S. Isolated noncompaction of the ventricular myocardium: ultrafast computer tomography and magnetic resonance imaging. Int J Cardiovasc Imaging. $2001 ; 17: 305-14$

15. Junga G, Kneifel S, Von Smekal A, Steinert H, Bauersfeld U. Myocardial ischaemia in children with isolated ventricular non-compaction. Eur Heart J. 1999; 20: 910-16.

16. Ichida F, Tsubata S, Bowles KR, Haneda N, Uese K, Miyawaki T. Novel gene mutations in patients with left ventricular noncompaction or Barth syndrome. Circulation. 2001; 103: 1256-63.

17. Elias J, Valadão W, Kuniyoshi R, Queiroz A, Peixoto CA. Miocárdio não compactado isolado. Arq Bras Cardiol. 2000; 74: 253-7.

18. Conraads V, Paelinck B, Vorlat A, Vorloit A, Goethals M, Jacobs W. Isolated non-compaction of the left ventricle: a rare indication for transplantation. J Heart Lung Transplant. 2001; 20: 904-7. 